Gate-dependent tunneling-induced level shifts observed in carbon nanotubes quanum dots

Holm, Jeppe Vilstrup; Jørgensen, Henrik Ingerslev; Grove-Rasmussen, Kasper; Paaske, Jens; Flensberg, Karsten; Lindelof, Poul Erik

Published in:

Physical Review B. Condensed Matter and Materials Physics

DOI:

10.1103/PhysRevB.77.161406

Publication date:

2008

Document version

Early version, also known as pre-print

Citation for published version (APA):

Holm, J. V., Jørgensen, H. I., Grove-Rasmussen, K., Paaske, J., Flensberg, K., \& Lindelof, P. E. (2008). Gatedependent tunneling-induced level shifts observed in carbon nanotubes quanum dots. Physical Review $B$. Condensed Matter and Materials Physics, 77(16), 161406. https://doi.org/10.1103/PhysRevB.77.161406 


\title{
Gate-dependent tunneling-induced level shifts observed in carbon nanotube quantum dots
}

\author{
J. V. Holm, H. I. Jørgensen, K. Grove-Rasmussen, J. Paaske, K. Flensberg, and P. E. Lindelof \\ Niels Bohr Institute and Nano-Science Center, University of Copenhagen, DK-2100 Copenhagen, Denmark
}

(Received 12 March 2008; published 25 April 2008)

\begin{abstract}
We have studied electron transport in clean single-walled carbon nanotube quantum dots. Because of the large number of the Coulomb blockade diamonds simultaneously showing both shell structure and the Kondo effect, we are able to perform a detailed analysis of tunneling renormalization effects. Thus, determining the environment induced level shifts of this artificial atom. In shells where only one of the two orbitals is strongly coupled, we observe a marked asymmetric gate dependence of the inelastic cotunneling lines together with a systematic gate dependence of the size (and shape) of the Coulomb diamonds. These effects are all given a simple explanation in terms of second-order perturbation theory in the tunnel coupling.
\end{abstract}

DOI: 10.1103/PhysRevB.77.161406

PACS number(s): 73.63.Kv, 72.10.Fk

A solid-state quantum dot (QD) is often referred to as an artificial atom, ${ }^{1,2}$ and the energy levels of this artificial atom can be measured by using a bias spectroscopy in the Coulomb blockade regime. ${ }^{3-6}$ In this regime, the couplings to the metallic leads are usually regarded as small perturbations, giving rise to a finite conductance without otherwise affecting the internal structure and energy levels of the QD. Deviations from the perfect shell structure in, for example, carbon nanotubes are usually attributed to the imperfections of the single-walled carbon nanotube (SWCNT). Consequences of stronger tunnel coupling to the metallic leads have been studied in the context of broadening of the energy levels ${ }^{7}$ and the Kondo effects. ${ }^{8-10}$

For moderate coupling strengths, a certain degree of level shifts and broadening is expected to arise from the hybridization with the leads. Nevertheless, these effects are difficult to distinguish and therefore rarely discussed in the literature. So far, the only case where such renormalization effects have been shown to have important observable consequences is for QD spin-valve systems, where either noncollinear magnetization gives rise to off-diagonal renormalization of the spin structure on the QD ${ }^{11}$ or spin-dependent tunneling gives rise to a gate-dependent exchange field on the QD. ${ }^{12,13}$ In a similar manner, one might expect a difference in couplings to the two orbitals (or subbands) in the SWCNT to give rise to distinct observable renormalization effects in a SWCNT.

In this Rapid Communication, we report on measurements on several SWCNT devices showing regular four-electron shell structure. The best device shows a total of 60 regular four-electron shells, allowing for a unique quantitative and statistical analysis of renormalization effects. In particular, we study the cases where the four-electron shells exhibit the Kondo effect in only one of the diamonds in the quartet, indicating that one of the (almost) degenerate orbitals has a stronger coupling to the leads than the other. Such behavior is seen in 28 four-electron shells. Based on this analysis, we find that the relative magnitudes of the tunnel coupling of orbitals 1 and 2 determine three distinctly different types of quartets.

A difference in tunnel coupling for the two orbitals gives rise to two features: (1) the inelastic cotunneling threshold given by the orbital splitting develops a marked gate dependence and (2) the addition energies of the individual Coulomb diamonds within a quartet deviate from the constant- interaction model, ${ }^{2}$ depending on the relative magnitude of the tunneling couplings. Both features can be explained by the level shifts of the many-body states of the QD, which are calculated here to second order in the tunnel coupling.

The devices were made on a highly $n$-doped $500 \mu \mathrm{m}$ thick silicon substrate with a $500 \mathrm{~nm}$ thick $\mathrm{SiO}_{2}$ top layer. Chemical vapor deposition was used to grow the SWCNTs from the predefined catalyst particle areas. The SWCNTs were contacted by thermal evaporation of titanium/ aluminum/titanium $(5 \mathrm{~nm} / 40 \mathrm{~nm} / 5 \mathrm{~nm}),{ }^{21}$ and the electrodes were separated by $300 \mathrm{~nm}$ and patterned by electron beam lithography. The silicon substrate was used as a back gate to change the electrostatic potential of the SWCNT QD. The electron transport measurements were carried out in a cryogenic insert system with a base temperature of $300 \mathrm{mK}$. Two-terminal conductance was measured by using lock-in techniques with $13 \mu \mathrm{V}$ ac excitation amplitude and a dc bias voltage $\left(V_{s d}\right)$ applied to the source, while the drain was grounded through a current amplifier.

Room temperature measurements of the conductance as a function of back gate voltage $\left(V_{g}\right)$ indicate that the SWCNT



FIG. 1. Bias spectroscopy plot of a SWCNT QD for $-10 \mathrm{~V}<V_{g}<10 \mathrm{~V}$. The Coulomb diamonds are seen for almost every added electron (285), and 88 odd-occupancy Coulomb blockade diamonds exhibit a zero-bias Kondo resonance. 
in the presented device is metallic. In the full gate voltage span, $-10 \mathrm{~V}<V_{g}<10 \mathrm{~V}$, four-electron shell filling is observed at low temperatures, and an overview of the regular behavior of the device can be seen in Fig. 1. In total, 60 full four-electron shells are observed across the measured gate voltage range. Disregarding a few gate switches, we have observed 285 consecutive electron additions. The 60 fourelectron shells can be identified, the remaining 45 electrons are counted in incomplete shells. We have extracted the Kondo temperatures from 88 equilibrium Kondo resonances from the width of the Kondo peak $\left(W_{F W H M} \sim 4 k_{B} T_{K}\right) .{ }^{14}$ The Kondo temperatures found by measuring the Kondo peak widths are in good agreement with Kondo temperatures found for selected peaks, when measuring the zero-bias conductance as a function of temperature and fitting to the universal scaling function ${ }^{15}$ (not shown). We observe no systematic gate dependence of the Kondo temperatures, with an average of $\left\langle T_{K}\right\rangle \approx 1.2 \mathrm{~K}$ and a standard deviation of $0.5 \mathrm{~K}$.

A more detailed bias spectroscopy plot of one of the fourelectron shells from Fig. 1 can be seen in Fig. 2(a). The plot resembles the previously reported characteristics of a SWCNT QD in the Kondo regime. ${ }^{8,9}$ In diamonds $N+1$ and $N+3$, corresponding to odd-electron occupation, spin-1/2 Kondo ridges can be seen as horizontal lines at zero-bias voltage. In the $N+1, N+2$, and $N+3$ diamonds, the onset of inelastic cotunneling can be seen as practically horizontal lines at $V_{s d} \approx \pm 0.7 \mathrm{mV} .^{16}$ The cotunneling ridge at $V_{s d} \approx+0.7 \mathrm{mV}$ in the $N+2$ diamond is significantly Kondo enhanced. ${ }^{10}$ Equilibrium and nonequilibrium Kondo features are observed in the full gate voltage span. From the plot, we can extract the characteristic energies of the system as indicated in the schematic drawing on the right in Fig. 2(a).,17 We obtain a Coulomb energy of $U=2.9 \mathrm{meV}$, orbital splitting $\delta=0.7 \mathrm{meV}$, and single-particle level spacing $\Delta E=3.5 \mathrm{meV}$.

In addition to this well-established four-electron shell behavior, we have observed two distinctly different types of four-electron shells, which are exemplified by the plots in Figs. 2(b) and 2(c). In Fig. 2(b), there is a Kondo ridge in $N+3$, but the expected Kondo ridge in the $N+1$ diamond is absent. In addition, the cotunneling thresholds in the $N+1$ and $N+2$ diamonds increase from left $\left(V_{s d} \approx \pm 0.4 \mathrm{mV}\right)$ to right $\left(V_{s d} \approx \pm 1.2 \mathrm{mV}\right)$, showing almost identical gate dependence. In Fig. 2(c), the situation is reversed: A zero-bias Kondo ridge now only occurs in the $N+1$ diamond, and the cotunneling thresholds in the N+2 and N+3 diamonds decrease from left $\left(V_{s d} \approx \pm 0.4 \mathrm{mV}\right)$ to right $\left(V_{s d} \approx 0 \mathrm{mV}\right)$. The electron-hole symmetry (around the middle of the $N+2$ diamond $^{6}$ ) which is observed in panel (a) is clearly lifted in panels (b) and (c). Furthermore, the main features in panels (b) and (c) are each others mirror image.

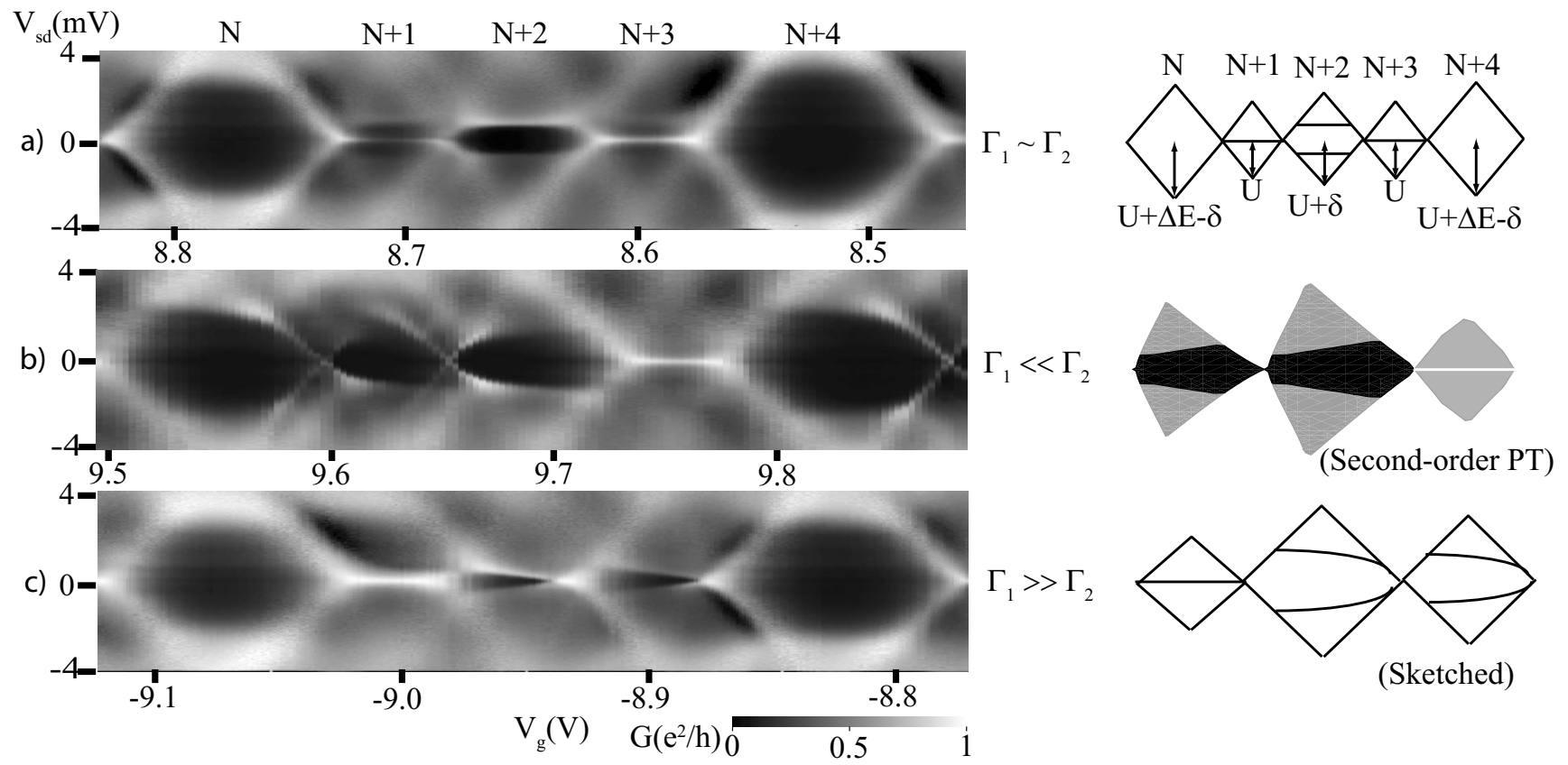

FIG. 2. Differential conductance as a function of bias voltage $V_{s d}$ and gate voltage $V_{g}$ (left). Schematic of the four-electron shells (right). (a) Four-electron shell filling for $\Gamma_{1} \sim \Gamma_{2}$, with strong enough coupling to facilitate Kondo ridges in the $N+1$ and $N+3$ diamonds, as shown in the schematic. A total of 17 four-electron shells show this type of behavior across the full gate voltage range. (b) Four-electron shell filling for $\Gamma_{1} \ll \Gamma_{2}$. In the $N+1$ and $N+2$ diamonds, the inelastic cotunneling threshold has clearly acquired a gate dependence. In total, there are 21 four-electron shells showing this type of behavior. Here, the right panel shows the cotunneling thresholds and diamond edges as calculated within second-order PT (the white Kondo ridge is drawn by hand). (c) Four-electron shell filling for $\Gamma_{1} \gg \Gamma_{2}$. Gate-dependent cotunneling thresholds are now observed in the N+2 and N+3 diamonds instead. In total, there are 7 four-electron shells showing this type of behavior. 15 four-electron shells could not be uniquely categorized. In the 28 shell sequences where $\Gamma_{1} \gg \Gamma_{2}$ or $\Gamma_{1} \ll \Gamma_{2}$, the gatedependent cotunneling threshold ridges inside the diamonds appear to form a $U$ or $V$ lying down with the openings facing the zero-bias Kondo ridge. Together the two $U$ 's $(V$ 's) and the Kondo ridge resemble a two-headed arrow, where the $U(V)$ form the two heads and the Kondo ridge the shaft. This means that the tree center diamonds in the different domains have cotunneling and Kondo ridges, which can be schematically summarized as $\Gamma_{1} \sim \Gamma_{2}:-=-, \Gamma_{1} \ll \Gamma_{2}:<<-$, and $\Gamma_{1} \gg \Gamma_{2}:->>$. 


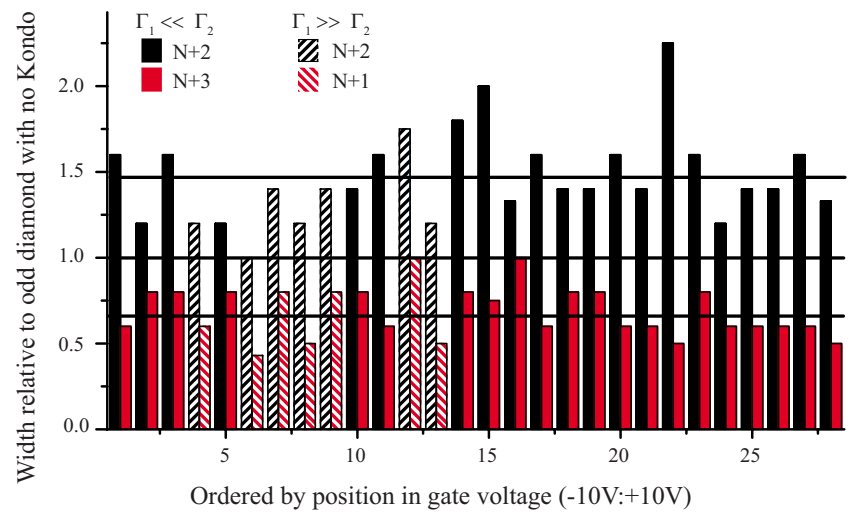

FIG. 3. (Color online) The ratio of the widths of the diamonds (proportional to the additional energies) in the 28 unusual fourelectron shells to the odd-electron diamonds without a Kondo ridge in the same four-electron shells. The solid (cross-hatched) pairs is for $\Gamma_{1} \ll \Gamma_{2}\left(\Gamma_{1} \gg \Gamma_{2}\right)$. The average width of the even-electron diamonds (black) is 1.47, and the average width of the odd-electron diamonds with a Kondo ridge [red (dark gray)] is 0.66 , as indicated by the black lines.

As already mentioned, the sample shows a large number of four-electron shells (60). There are 28 of them that show only a single equilibrium Kondo resonance and exhibit gatedependent inelastic cotunneling lines inside the two adjacent Coulomb diamonds.

Furthermore, there is a clear correlation between the size of the odd-electron diamonds with and without the zero-bias Kondo ridge, respectively. This is demonstrated in Fig. 3, where the widths of the $N+2$ diamonds (black) and the oddelectron diamonds, which exhibit a Kondo resonance [red (dark gray)], are shown relative to the widths of the oddelectron diamonds not showing a Kondo resonance. For all 28 quartets of this type, the diamond showing a zero-bias Kondo ridge is narrower than the diamond without the Kondo ridge, which is independent of whether $\Gamma_{1} \ll \Gamma_{2}$ or $\Gamma_{1} \gg \Gamma_{2}$. Observing this in 28 out of 60 shell sequences, this behavior cannot be dismissed as a measurement irregularity. We have seen similar gate dependences in several other SWCNT devices with intermediate coupling to the electrodes.

We shall now focus on the quartet mapped out in Fig. 2 (b), where $\Gamma_{1} \ll \Gamma_{2}$. The presence and the absence of a zerobias Kondo ridge in the $N+3$ and $N+1$ diamonds, respectively, already indicate that the two orbitals must have different tunnel couplings, since the Kondo temperatures must be, respectively, larger than or smaller than the base temperature $(300 \mathrm{mK})$. The Kondo temperatures are given by $T_{K}^{(i)} \sim \sqrt{4 \Gamma_{i} U} \exp \left(-\pi U /\left(8 \Gamma_{i}\right)\right)$ (Ref. 18) in terms of the individual tunnel broadenings $\Gamma_{i}=\sum_{\alpha=s, d} \Gamma_{i, \alpha}$, with $\Gamma_{i, \alpha}$ $=\pi \nu_{F}\left|t_{i, \alpha}\right|^{2}$, determined by the density of states in the source and drain electrodes $\nu_{F}$, and their respective tunnel couplings to the dot $t_{i, \alpha}$ assumed to be energy independent in the relevant energy range. The lack of particle-hole symmetry in this quartet is thus consistent with the orbital symmetry being lifted by a difference between $\Gamma_{1}$ and $\Gamma_{2}$. The $\Gamma_{i}$ are generally difficult to extract, but by fitting the even valley part of the Coulomb peaks of Fig. 2(b) to a Lorentzian (cf.

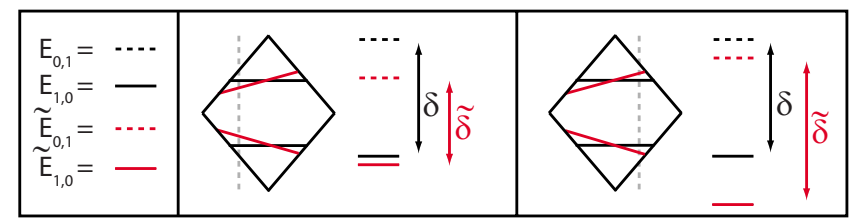

FIG. 4. (Color online) Schematic drawing illustrating the difference in renormalization of $\widetilde{E}_{1,0}$ [red (dark gray) solid lines $]$ and $\widetilde{E}_{0,1}$ [red (dark gray) dashed lines] at opposite ends of the $N+1$ diamond. Assuming $\Gamma_{1} \ll \Gamma_{2}$, the $(0,1)$ level is strongly perturbed by virtual emptying (left), whereas the $(1,0)$ level is most strongly perturbed by virtual double occupancy (right).

Ref. 19), we estimate that $\Gamma_{1} \approx 0.08 \mathrm{meV}$ and $\Gamma_{2} \approx 0.46$ meV. This yields $T_{K}^{(1)} \sim 7 \mu \mathrm{K}$ and $T_{K}^{(2)} \sim 2 \mathrm{~K}$, consistent with the observation of a Kondo ridge only in the $N+3$ diamond at $T \sim 300 \mathrm{mK}$.

The finite-bias cotunneling lines inside the $N+1$ and $N+2$ diamonds of Fig. 2(b) can be ascribed to an inelastic cotunneling processes in which an electron traversing the dot from source to drain has enough energy to excite a single electron from orbital 1 to orbital 2. The individual manybody states of the dot electrons are renormalized by virtual charge fluctuations, and, as we shall argue below, these tunneling-induced level shifts can indeed give rise to a gatedependent cotunneling threshold.

Within a single four-electron shell, the many-body states of the dot electrons can be enumerated by the number of electrons occupying the two highest lying single-particle orbitals. In terms of bare level position $\varepsilon_{d}$, intradot Coulomb repulsion $U$, and orbital splitting $\delta$, the corresponding energies are given by $E_{i, j}=(i+j) \varepsilon_{d}+j \delta+(i+j)(i+j-1) U / 2$, where $i(j)$ denotes the number of electrons in orbital 1(2). The level shifts, $\delta E_{i, j}=\widetilde{E}_{i, j}-E_{i, j}$, are determined within second-order many-body perturbation theory (PT) in the tunnel coupling by considering all possible fluctuations experienced by a given charge configuration. ${ }^{20}$ For example, the charge state $|1,0\rangle$ connects to $|0,0\rangle$ and to $|2,0\rangle$ and $|1,1\rangle$, respectively, by virtual tunneling out and in of an electron. This leads to the following shift in energy:

$$
\begin{aligned}
\delta E_{1,0}= & \sum_{\alpha=s, d} \int_{-D}^{D} \frac{d \omega}{\pi} \operatorname{Re}\left[\frac{\Gamma_{1 \alpha}\left[1-f\left(\omega-\mu_{\alpha}\right)\right]}{E_{1,0}-\left(E_{0,0}+\omega\right)+i \Gamma}\right. \\
& \left.+\frac{\Gamma_{1 \alpha} f\left(\omega-\mu_{\alpha}\right)}{E_{1,0}+\omega-E_{2,0}+i \Gamma}+\frac{2 \Gamma_{2 \alpha} f\left(\omega-\mu_{\alpha}\right)}{E_{1,0}+\omega-E_{1,1}+i \Gamma}\right],
\end{aligned}
$$

where $2 D$ is the conduction-electron bandwidth, $f(\omega)$ is the Fermi function, and $\mu_{s, d}$ are the chemical potentials of the source and drain electrodes. A level broadening of order $\Gamma$ $\sim \Gamma_{1}+\Gamma_{2}$ has been included in the energy denominators and serves to cut off the otherwise logarithmically singular result. Notice that the last process has multiplicity two from spin and that only this process has the amplitude $\Gamma_{2}$. The excited state is renormalized correspondingly and $\delta E_{0,1}$ is given by Eq. (1) with all orbital indices reversed, i.e., $E_{i, j} \rightarrow E_{j, i}$ and $\Gamma_{1 \alpha} \leftrightarrow \Gamma_{2 \alpha}$. This means that for gate voltages close to the $0 / 1$ (left diamond corner) or $1 / 2$ (right diamond corner) charge- 
degeneracy point, respectively, it is the first or the two last terms in Eq. (1) which dominate the renormalization, with small energy denominators. When $\Gamma_{1} \ll \Gamma_{2}$, the $E_{0,1}$ energy level (orbital 2) will have a large shift at the left corner of the diamond (0/1) and the $E_{1,0}$ energy level (orbital 1) will have a large shift at the right corner $(1 / 2)$, as illustrated in Fig. 4. This means that the cotunneling threshold increases from left to right when $\Gamma_{1} \ll \Gamma_{2}$ and decreases from left to right when $\Gamma_{1} \gg \Gamma_{2}$. To be more precise, the inelastic cotunneling threshold inside the $N+1$ diamond is determined as the energy $\tilde{\delta}$ solving the equation $\widetilde{\delta}=\widetilde{E}_{1,0}-\widetilde{E}_{0,1}$, with $\mu_{s}-\mu_{d}= \pm \tilde{\delta} / e$ in the expressions for the $\widetilde{E}_{i, j}$. The solution, with parameters found above, ${ }^{22}$ is plotted in the right panel of Fig. 2(b), with a reasonable resemblance to the gate dependence observed in the left panel. Expanding near the middle of the $N+1$ diamond, we find that $d \tilde{\delta} / d \varepsilon_{d} \approx-4\left(\Gamma_{2}-\Gamma_{1}\right) /(\pi U)$ for $\varepsilon_{d}=-U / 2$. Thus, if $\Gamma_{2}>\Gamma_{1}$, the cotunneling threshold clearly increases with gate voltage $\left(e V_{g} \propto-\varepsilon_{d}\right)$, i.e., in the direction toward the stronger coupled $N+3$ diamond, as we observe.

Calculating all the relevant level shifts, one can also determine the changed gate voltage width $\Delta V_{g}(n)$ of the $N+n$ diamond. We find that $\Delta V_{g}(1) \approx U-\left(2 \Gamma_{1} / \pi\right) \ln (U / \Gamma)$, $\Delta V_{g}(2) \approx U+\delta$, and $\Delta V_{g}(3) \approx U-\left(2 \Gamma_{2} / \pi\right) \ln (U / \Gamma)$, which are strictly valid only in the regime where $\delta \ll \Gamma \ll U$. A larger $\delta$ implies more correction terms, but the main trend remains the same: a large $N+2$ diamond and a small $N+3$ diamond, as compared to the $N+1$ diamond $\left(\Gamma_{1} \ll \Gamma_{2}\right)$ in agreement with the statistics presented in Fig. 3.

Finally, we have calculated the shifted Coulomb diamond boundaries by using the second-order level shifts. For the found parameters, this gives rise to the diamond boundaries seen in the right panel of Fig. 2(b). The details of this figure are not entirely reliable, and more work is needed in order to proceed beyond second-order PT and to incorporate the nonequilibrium mixing of ground and excited states within each diamond. Nevertheless, this simple analysis indicates that tunneling-induced level shifts may give rise to skewed (i.e., nonparallel edges), rather than merely shifted and rescaled Coulomb diamonds, which is, in fact, seen in several of our experimental quartets, e.g., Fig. 2(c).

In conclusion, we have fabricated gated SWCNT devices with intermediate transparency contacts. Transport measurements have revealed three distinct types of four-electron shells, two of which show a marked gate dependence of the inelastic cotunneling ridges, together with a systematic pattern for the individual diamond widths within a shell. Calculating second-order level shifts for the many-body states on the QD, we have explained all of these observations in terms of a difference in tunnel coupling to the two orbitals in the SWCNT.

We acknowledge support from EU projects ULTRA-1D, SECOQC, CARDEQ, and the Danish Research Council.
${ }^{1}$ L. P. Kouwenhoven, T. H. Oosterkamp, M. W. S. Danoesastro, M. Eto, D. G. Austing, T. Honda, and S. Tarucha, Science 278, 1788 (1997).

${ }^{2}$ R. Hanson, L. P. Kouwenhoven, J. R. Petta, S. Tarucha, and L. M. K. Vandersypen, Rev. Mod. Phys. 79, 1217 (2007).

${ }^{3}$ S. Tans, M. H. Devoret, H. Dai, A. Thess, R. E. Smalley, L. J. Geerlings, and C. Dekker, Nature (London) 386, 474 (1997).

${ }^{4}$ M. Bockrath, D. H. Cobden, P. L. McEuen, N. G. Chopra, A. Zettl, A. Thess, and R. E. Smalley, Science 275, 1922 (1997).

${ }^{5}$ D. H. Cobden and J. Nygård, Phys. Rev. Lett. 89, 046803 (2002).

${ }^{6}$ P. J. Herrero, S. Sapmaz, C. Dekker, L. P. Kouwenhoven, and H. S. van der Zandt, Nature (London) 429, 389 (2004).

${ }^{7}$ W. Liang, M. Bockrath, and H. Park, Phys. Rev. Lett. 88, 126801 (2002).

${ }^{8}$ J. Nygård, D. H. Cobden, and P. E. Lindelof, Nature (London) 408, 342 (2000).

${ }^{9}$ B. Babic, T. Kontos, and C. Schönenberger, Phys. Rev. B 70, 235419 (2004).

${ }^{10}$ J. Paaske, A. Rosch, P. Wölfe, N. Mason, C. M. Marcus, and J. Nygård, Nat. Phys. 2, 460 (2006).

${ }^{11}$ S. Braig and P. W. Brouwer, Phys. Rev. B 71, 195324 (2005).

${ }^{12}$ J. Martinek, M. Sindel, L. Borda, J. Barnas, R. Bulla, J. Konig,
G. Schon, S. Maekawa, and J. von Delft, Phys. Rev. B 72, 121302(R) (2005).

${ }^{13}$ J. R. Hauptmann, J. Paaske, and P. E. Lindelof, Nature Physics, doi:10.1038/nphys931.

${ }^{14}$ T. Micklitz, A. Altland, T. A. Costi, and A. Rosch, Phys. Rev. Lett. 96, 226601 (2006).

${ }^{15}$ T. A. Costi, A. C. Hewson, and V. Zlatic, J. Phys.: Condens. Matter 6, 2519 (1994).

${ }^{16}$ S. De Franceschi, S. Sasaki, J. M. Elzerman, W. G. van der Wiel, S. Tarucha, and L. P. Kouwenhoven, Phys. Rev. Lett. 86, 878 (2001).

${ }^{17}$ Y. Oreg, K. Byczuk, and B. I. Halperin, Phys. Rev. Lett. 85, 365 (2000).

${ }^{18}$ A. C. Hewson, The Kondo Problem to Heavy Fermions (Cambridge University Press, Cambridge, 1993).

${ }^{19}$ H. I. Jørgensen, T. Novotny, K. Grove-Rasmussen, K. Flensberg, and P. E. Lindelof, Nano Lett. 7, 2441 (2007).

${ }^{20}$ F. D. M. Haldane, Phys. Rev. Lett. 40, 416 (1978).

${ }^{21}$ The sample was cooled to $30 \mathrm{mK}$, but due to a thin oxide layer forming between the titanium and aluminum during evaporation, no superconducting gap has been observed.

${ }^{22}$ As a rough estimate, we use $\delta$ and $U$ found from Fig. 2(a). 